\title{
SEMI-HYPERBOLIC PATCHES ARISING FROM A TRANSONIC SHOCK IN SIMPLE WAVES INTERACTION
}

\author{
Kyungwoo SONG
}

\begin{abstract}
In this paper we consider a Riemann problem, in particular, the case of the presence of the semi-hyperbolic patches arising from a transonic shock in simple waves interaction. Under this circumstance, we construct global solutions of the two-dimensional Riemann problem of the pressure gradient system. We approach the problem as a Goursat boundary value problem and a mixed initial-boundary value problem, where one of the boundaries is the transonic shock.
\end{abstract}

\section{Introduction}

We consider a semi-hyperbolic region formed by the interaction of simple waves, which occurs frequently in the Riemann problem of the pressure gradient system

$$
\left\{\begin{array}{r}
u_{t}+p_{x}=0, \\
v_{t}+p_{y}=0, \\
E_{t}+(u p)_{x}+(v p)_{y}=0
\end{array}\right.
$$

in two space dimensions, where $E=p+\left(u^{2}+v^{2}\right) / 2$. See the book of Li et al. [3] or $[5,8,9,10]$ for more information on the system. In the self-similar coordinates $(\xi, \eta)=(x / t, y / t)$, the pressure gradient system has a second-order equation for $p$ of the form

(2) $\left(p-\xi^{2}\right) p_{\xi \xi}-2 \xi \eta p_{\xi \eta}+\left(p-\eta^{2}\right) p_{\eta \eta}+\frac{\left(\xi p_{\xi}+\eta p_{\eta}\right)^{2}}{p}-2\left(\xi p_{\xi}+\eta p_{\eta}\right)=0$,

which transforms into

$$
\left(p-r^{2}\right) p_{r r}+\frac{p}{r^{2}} p_{\theta \theta}+\frac{p}{r} p_{r}+\frac{1}{p}\left(r p_{r}\right)^{2}-2 r p_{r}=0,
$$

Received June 12, 2012.

2010 Mathematics Subject Classification. Primary 35L65, 35J70, 35R35.

Key words and phrases. 2-D Riemann problem, characteristic decomposition, simple waves, transonic shocks.

This research was supported by Basic Science Research Program through the National Research Foundation of Korea (NRF) funded by the Ministry of Education, Science and Technology (NRF-2010-0011541). 


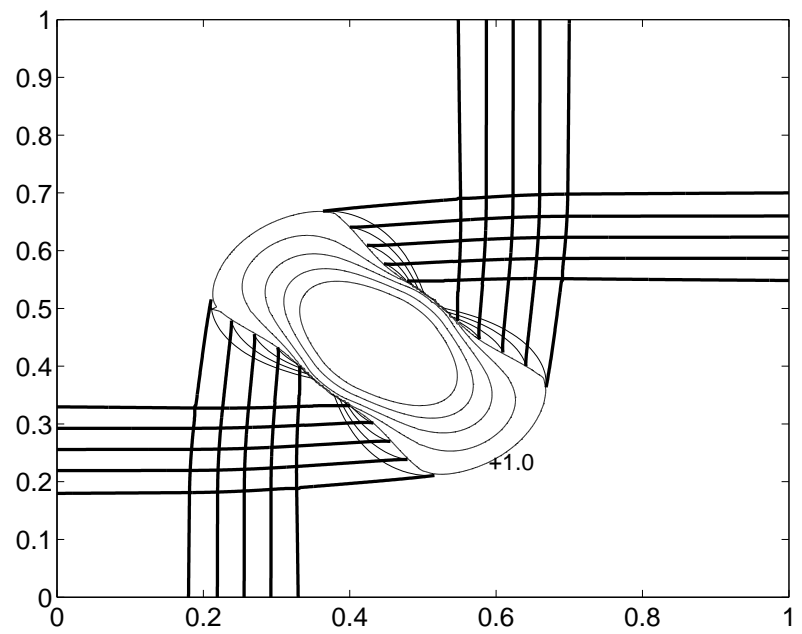

Figure 1. Four semi-hyperbolic patches, marked by the short light curves and overlapped by some bold ones, in the interaction of two forward and two backward rarefaction waves for the Euler system with a gas constant $\gamma=1.4$. The five closed curves at the center are contour curves of the pseudo-Mach number, which mark the subsonic region; the bold curves originating from the boundaries are characteristics; the short light curves in the semihyperbolic regions are also characteristics. The solution has two axes of symmetry (courtesy of Glimm et al. [2]).

or

$$
p_{\theta \theta}-\frac{r^{2}\left(r^{2}-p\right)}{p} p_{r r}+\frac{r p_{r}}{p}\left(p+\frac{r^{3} p_{r}}{p}-2 r^{2}\right)=0
$$

in polar coordinates $(r, \theta)$ of the $(\xi, \eta)$-plane.

As the configuration $G$ in [3], in this paper we consider the case of the presence of the semi-hyperbolic patches with a transonic shock in a simple wave environment. The regions identified by the existence of a family of characteristics that start on sonic curves and end on transonic shock curves are called semi-hyperbolic patches. These regions are different from the typical fully hyperbolic regions. As in the result of [2], in several numerical calculations, we frequently encounter this kind of regions in several configurations in the twodimensional Riemann problems of Euler systems as well as pressure gradient systems (see Figure 1). We believe that it is helpful and essential to understand these patches of solutions locally before resolving the Riemann problems globally. A global solution to the boundary value problem of the Riemann problem of a hyperbolic system of equations can be patched together by pieces along characteristic lines, sonic curves, shock waves or other natural boundaries. We 
attempt in this paper to construct the patch in a way that is sufficient for understanding the internal structure of the patch, while postponing its connection to the subsonic domain for later work. In other words, we construct a number of these patches in anticipation for further cutting or rubbing on the transonic edges to fit to the subsonic domain, and focus on which key curve can determine such a patch.

In the self-similar plane, the pressure gradient system becomes

$$
\left\{\begin{array}{l}
-\xi u_{\xi}-\eta u_{\eta}+p_{\xi}=0 \\
-\xi v_{\xi}-\eta v_{\eta}+p_{\eta}=0 \\
-\xi E_{\xi}-\eta E_{\eta}+(p u)_{\xi}+(p v)_{\eta}=0 .
\end{array}\right.
$$

Let $\Sigma:=\{\eta=\eta(\xi)\}$ be a given smooth transonic shock with slope $\sigma=\eta^{\prime}(\xi)$ appearing in the interaction of rarefaction waves or simple waves. The shock locates between an elliptic region and a simple wave zone, denoted by $R$. Then the shock location is determined by the famous Rankine-Hugoniot condition

$$
\sigma=\frac{d \eta}{d \xi}=\frac{\xi \eta \pm \sqrt{\bar{p}\left(\xi^{2}+\eta^{2}-\bar{p}\right)}}{\xi^{2}-\bar{p}}
$$

or equivalently, in polar coordinate system $(r, \theta)$, it becomes

$$
r^{\prime}(\theta)= \pm r \sqrt{\frac{r^{2}-\bar{p}}{\bar{p}}}
$$

where $r=\sqrt{\xi^{2}+\eta^{2}}, \tan \theta=\eta / \xi$, and $\bar{p}$ is the average value of $p$ between two neighboring states of the shock. In this paper, we concern about the positive derivative case of $r^{\prime}$.

The second-order equation (3) can be decomposed into

$$
\partial_{+} \partial_{-} p=q\left(\partial_{+} p-\partial_{-} p\right) \partial_{-} p, \quad \partial_{-} \partial_{+} p=q\left(\partial_{-} p-\partial_{+} p\right) \partial_{+} p,
$$

where

$$
q:=\frac{r^{2}}{4 p\left(r^{2}-p\right)}
$$

and

$$
\partial_{+}=\partial_{\theta}+\lambda^{-1} \partial_{r}, \quad \partial_{-}=\partial_{\theta}-\lambda^{-1} \partial_{r}, \quad \lambda^{-1}=\sqrt{r^{2}\left(r^{2}-p\right) / p} .
$$

Here, $\partial_{+} p, \partial_{-} p$ are the directional derivatives of $p$ along the positive and the negative characteristics, respectively. We also note that equation (3) transforms into the system of equations

(8) $\left(\begin{array}{c}\partial_{+} p \\ \partial_{-} p \\ p\end{array}\right)_{\theta}+\left(\begin{array}{ccc}-\frac{1}{\lambda} & 0 & 0 \\ 0 & \frac{1}{\lambda} & 0 \\ 0 & 0 & 0\end{array}\right)\left(\begin{array}{c}\partial_{+} p \\ \partial_{-} p \\ p\end{array}\right)_{r}=\left(\begin{array}{c}q\left(\partial_{-} p-\partial_{+} p\right) \partial_{+} p \\ q\left(\partial_{+} p-\partial_{-} p\right) \partial_{-} p \\ \frac{1}{2}\left(\partial_{+} p+\partial_{-} p\right)\end{array}\right)$.

Then there are 3 eigenvalues

$$
\lambda_{1}=\lambda^{-1}, \quad \lambda_{2}=0, \quad \lambda_{3}=-\lambda^{-1}
$$




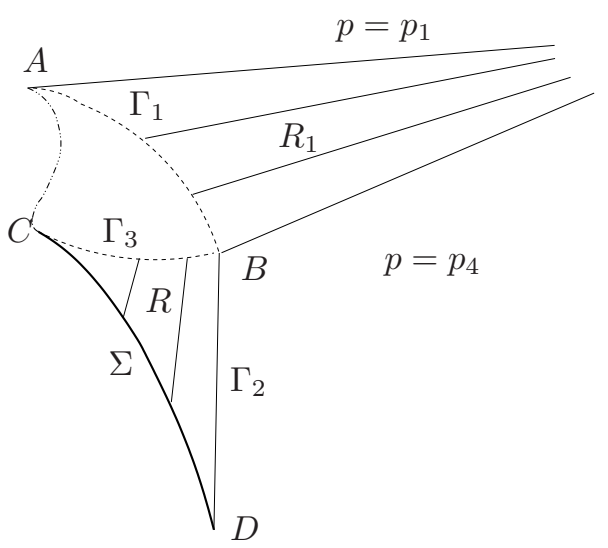

Figure 2. Interaction of two simple waves $R_{1}$ and $R . A C$ is a sonic curve.

and their corresponding 3 eigenvectors

$$
l_{1}=(0,1,0), \quad l_{2}=(0,0,1), \quad l_{3}=(1,0,0) .
$$

In [6], Song and Zheng obtained the global solution of semi-hyperbolic patches under the assumption that there is a negative characteristic $Y_{-}$emitting from a point, which is denoted by $X$ and located on the characteristic curve $A B$, to the region of $A B C$ (Figure 2). Then $Y_{-}$and the positive characteristic curve $A X$ are acting as two boundaries of the semi-hyperbolic region. In the paper, they started their exploration along the negative characteristic $Y_{-}$, which was set to understand a semi-hyperbolic patch of solutions. In other words, they considered global solutions in the region bounded by two characteristics emitting from $X$. It was also shown that there forms a transonic shock below the negative characteristic curve. In this paper, we approach the problem with a different and more natural view point. We regard the semi-hyperbolic patch as a result of a transonic shock. That is, in reverse, from a transonic shock, we show that there form a simple wave zone and then a semi-hyperbolic patch of the pressure gradient system. Therefore, in this paper we construct global solutions including the semi-hyperbolic patches obtained in [6] under more natural assumptions. The main contribution of this paper is to consider the semi-hyperbolic patches globally from the transonic shock to sonic lines.

Let $R_{1}$ be a simple wave zone connecting two constant states $p_{1}$, which is rescaled to 1 , and $p_{4}$. Denote the positive characteristic connecting points $B$ and $A$ by $\Gamma_{1}$, and the positive characteristic from $D$ to $B$ by $\Gamma_{2}$. Also, we denote the negative characteristic emitting from $B$ to the interaction zone by $\Gamma_{3}$. Then the following is the main result of this paper.

Main Theorem. For a given smooth shock $\Sigma$ and positive characteristics $\Gamma_{1}$ and $\Gamma_{2}$, we build a piecewise smooth solution of (8) satisfying the following property in a domain bounded by $\Sigma$ and $\Gamma_{1} \cup \Gamma_{2}$ : the solution is smooth in 
both the simple wave zone $R$ adjacent to the constant state $p=p_{4}$ and the interaction zone $S$ bounded by $\Gamma_{1}$ and $\Gamma_{3}$. However, the directional derivative of $p$ has a jump along the negative characteristic curve $\Gamma_{3}$.

In the following section, we first consider whether the negative characteristic from $B$ meets with the end of a given transonic shock and then construct global solutions in each simple wave and a semi-hyperbolic patch from the transonic shock to the sonic lines. We also provide a new maximum principle of solutions using a new decomposition of pressure gradient systems. This is simpler and clearer than the maximum principle in [6].

\section{Main result}

Let us approach this problem with a combination of two types: mixed initialboundary value problems and Goursat boundary value problems. Then we need to impose proper conditions of $\left(\partial_{+} p, \partial_{-} p, p\right)$ on the boundary $\Sigma \cup \Gamma_{1} \cup \Gamma_{2}$. First, it is known in [4] that the adjacent area to the constant state of a pressure gradient system is a simple wave zone, so it is very natural to impose the following condition of $\left(\partial_{+} p, \partial_{-} p, p\right)$ on $\Gamma_{2}$ if we take account of the adjacent constant state $p=p_{4}$ :

$$
p=p_{4}, \quad \partial_{+} p=0, \quad \text { and } \quad \partial_{-} p>0 .
$$

Second, we impose the condition of $\left(\partial_{+} p, \partial_{-} p, p\right)$ on the shock $\Sigma$ so that the region $R$ is a simple wave zone. To that end, we let $\left(\partial_{+} p, \partial_{-} p, p\right)$ satisfy the following properties:

- $p$ is smooth and increasing along the shock as $\theta$ increases. Here, this increasing property is related to the shape of the shock; refer to Remark 2.2 below;

- $\partial_{+} p=0$ on the shock to be compatible with the condition $\partial_{+} p=0$ along $\Gamma_{2}$;

- large enough $\partial_{-} p>0$ to satisfy the positivity of $r^{\prime}$ in (9).

Third, we need the following conditions at the point $B$ :

$$
p=p_{4}, \quad \partial_{+} p(B)>0, \quad \partial_{-} p(B)>0 .
$$

Fourth, let us impose the conditions of $\left(\partial_{+} p, \partial_{-} p, p\right)$ on $\Gamma_{1}$ so that

$$
\partial_{+} p>0, \quad \partial_{-} p>0,
$$

and $p$ is a proper increasing function along $\Gamma_{1}$. Here, $\partial_{-} p>0$ is given by the decomposition (6) with the initial condition at $B$. When we regard the given simple wave $R_{1}$ as the small perturbation from the planar wave case in [6], it is reasonable to have the condition $\partial_{+} p>0$. In the planar wave case, the fact that $\partial_{+} p>0$ can be obtained using the circular positive characteristic curve.

Remark 2.1. We assume that a negative characteristic $\Gamma_{3}$ emitting from the point $B$ meets with the sonic point $C$, which is the preassigned end point of the given shock. Otherwise, we have the following two cases regarding this issue. 
Case 1 (the negative characteristic $\Gamma_{3}$ meets with the shock at a point $C^{\prime}$ different from $C$ ). This is the same as the case when $\Gamma_{3}$ meets with the sonic point $C$. That is, a point $B^{\prime}$ connected to $C$ by a negative characteristic plays the role of $B$ by letting $\partial_{+} p=0$ on the curve $B B^{\prime}$ instead of $\partial_{+} p>0$.

Case 2 (the negative characteristic $\Gamma_{3}$ meets with a sonic point, say $C^{\prime \prime}$, different from $C$. See Figure 3). Let us fix one point $T \in C C^{\prime \prime}$. Since $\partial_{+} p=0$ along $\Gamma_{2}, \partial_{+} p=0$ along each negative characteristic emitting from $\Gamma_{2}$. So we see that $\partial_{+} p=0=\partial_{-} p$ at $T$. Owing to the decompositions (6), $\partial_{-} p=0$ along $Y_{+}$. Likewise, we see that $\partial_{-} p=0$ in the region, denoted by $\Omega_{0}$, formed by points $C, C^{\prime \prime}$, and $E$ in Figure 3. Furthermore, $\partial_{+} p=0=\partial_{-} p$ in the whole region $\Omega_{0}$. Hence $p:=p_{0}$ is constant along the curve $C C^{\prime \prime}$, that is, $C C^{\prime \prime}$ is a part of the circle $C_{1}: r^{2}=p_{0}$. However, we expect the solution to increase along the transonic shock and the sonic curve since the wave is in between two constant states with $p_{1}>p_{4}$. Furthermore, we obtain the zero gradient estimates of $p$ in the elliptic region of a free boundary problem near $C_{1} \cap C C^{\prime \prime}$, which is impossible. Therefore, we exclude this case (see Figure 3).

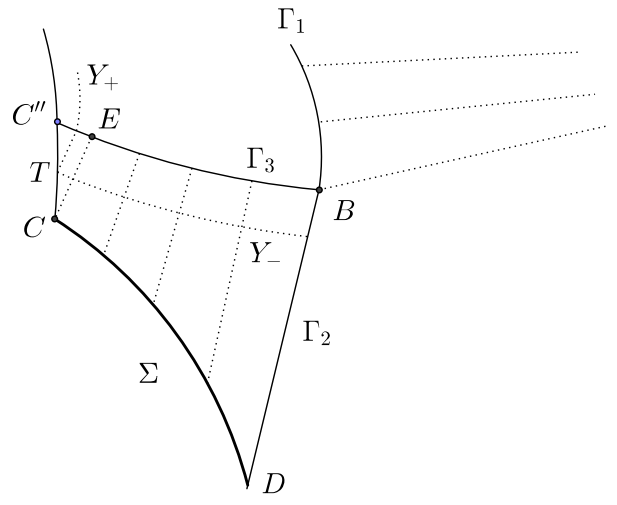

Figure 3. A negative characteristic $\Gamma_{3}$ meets with a sonic point

Let us define the directional derivative of $p$ along the shock $\Sigma:\{r=r(\theta)\}$ by

$$
\partial_{\Sigma} p:=\partial_{\theta} p+r^{\prime} \partial_{r} p
$$

in polar coordinate system. Let $\hat{p}$ be the value of $p$ on the subsonic region such that $\partial_{\Sigma} \hat{p}<0$ or $\left|\partial_{\Sigma} \hat{p}\right|$ is sufficiently small. In this paper, we are considering a transonic shock $\Sigma$ satisfying the following properties:

(S1) $r^{\prime}>0$ and $r^{\prime \prime}<0$ on $\Sigma$; 
(S2) at the point $D$ on $\Sigma$,

$$
\begin{aligned}
& r^{\prime}=\frac{\partial_{-} p-2 \partial_{\Sigma} p}{\partial_{-} p} \sqrt{\frac{r^{2}\left(r^{2}-p_{4}\right)}{p_{4}}}, \\
& r^{\prime}<\sqrt{\frac{r^{2}\left(r^{2}-p_{4}\right)}{p_{4}}} .
\end{aligned}
$$

The condition $(S 2)$ will be used for the compatibility condition of the local existence of solutions at $D$.

Remark 2.2 (A condition that guarantees $\partial_{\Sigma} p>0$ on $\Sigma$ ). Note from the $\mathrm{RH}$ condition (5), we have

Then

$$
p=-\hat{p}+\frac{2 r^{4}}{r^{2}+\left(r^{\prime}\right)^{2}} .
$$

$$
\partial_{\Sigma} p=-\partial_{\Sigma} \hat{p}+\frac{8 r^{5} r^{\prime}+16 r^{3}\left(r^{\prime}\right)^{3}-4 r^{4} r^{\prime} r^{\prime \prime}}{\left(r^{2}+\left(r^{\prime}\right)^{2}\right)^{2}} .
$$

We note $r^{\prime}>0$ and $r^{\prime \prime}<0$ by the assumption $(S 1)$. Hence for a subsonic solution $\hat{p}$ with $\partial_{\Sigma} \hat{p}<0$ or sufficiently small $\left|\partial_{\Sigma} \hat{p}\right|$,

$$
\partial_{\Sigma} p>0
$$

which implies $p$ is increasing along the shock from $D$ to $C$.

Letting $W=\left(\partial_{+} p, \partial_{-} p, p\right)^{T}$, we have the characteristic relation of (8) as follows:

$$
l_{k}\left(W_{\theta}+\lambda_{k} W_{r}\right)=b_{k},
$$

where $b_{k}$ is the inner product of $l_{k}$ with the vector in the right-hand side of (8). In the following lemma, we prove the existence of smooth solutions $W$ in the simple wave region $R$, where $p$ is constant along the positive characteristics.

Lemma 2.3 (Existence of smooth solutions in the region $R$ ). There exists a $C^{1}$ solution to the pressure gradient system (8) in the region $R \backslash\{C\}$.

Proof. [Local existence] For the local existence of solutions, let us approach the problem using the mixed initial-boundary value problem. The region of $R$ is bounded by two boundaries: one is the given shock and the other is a characteristic curve of the family $\lambda_{1}$. In fact, there are several methods to obtain the local existence of this type. In this paper let us use the method in $[7,10]$. For the local existence of smooth solutions for systems, we need the compatibility conditions at $D$. Define

$$
\phi^{A}(\theta):=W\left(\theta, r_{A}(\theta)\right)=\left(0, \partial_{-} p, p_{4}\right), \quad \phi^{B}(\theta):=W\left(\theta, r_{B}(\theta)\right)=\left(0, \partial_{-} p, p\right),
$$

where the given shock $\Sigma$ is represented by $r=r_{B}(\theta)$ and the given positive characteristic curve $\Gamma_{2}$ is represented by $r=r_{A}(\theta)$. Then at the point $D$,

$$
\begin{aligned}
& \partial_{+} \phi^{A}=W_{\theta}+\lambda_{1} W_{r}, \\
& \partial_{\Sigma} \phi^{B}=W_{\theta}+r_{B}^{\prime} W_{r} .
\end{aligned}
$$


From these relations, we determine $W_{\theta}(D)$ and $W_{r}(D)$. That is, for a smooth solution,

$$
\begin{aligned}
& W_{\theta}(D)=\frac{1}{r_{B}^{\prime}\left(\theta_{D}\right)-\lambda_{1}(D)}\left(r_{B}^{\prime}\left(\theta_{D}\right) \partial_{+} \phi^{A}(D)-\lambda_{1}(D) \partial_{\Sigma} \phi^{B}(D)\right), \\
& W_{r}(D)=\frac{1}{\lambda_{1}(D)-r_{B}^{\prime}\left(\theta_{D}\right)}\left(\partial_{+} \phi^{A}(D)-\partial_{\Sigma} \phi^{B}(D)\right) .
\end{aligned}
$$

Making use of the characteristic relations, we have

$$
\frac{1}{r_{B}^{\prime}-\lambda_{k}}\left(l_{k} \partial_{\Sigma} \phi^{B}-b_{k}\right)+\frac{1}{\lambda_{k}-\lambda_{1}}\left(l_{k} \partial_{+} \phi^{A}-b_{k}\right)=0, \quad k=2,3 .
$$

For $k=3, b_{3}=q\left(\partial_{-} p-\partial_{+} p\right) \partial_{+} p=0$ since $\partial_{+} p=0$ in the whole region $R$ including the boundary $\Sigma$ and $\Gamma_{2}$. In addition, $l_{3} \partial_{+} \phi^{A}=\partial_{+}\left(\partial_{+} p\right)=0$ on the curve $\Gamma_{2}$ and $l_{3} \partial_{\Sigma} \phi^{B}=\partial_{\Sigma}\left(\partial_{+} p\right)=0$ on the shock since $\partial_{+} p=0$ there. Hence the relation (13) holds.

For $k=2$, by a similar reasoning as in the case of $k=3$, we have

$$
\frac{1}{r_{B}^{\prime}\left(\theta_{D}\right)}\left(\partial_{\Sigma} p(D)-\frac{1}{2} \partial_{-} p(D)\right)+\frac{1}{2} \lambda(D) \partial_{-} p(D)=0,
$$

which holds at $D$ if the shock $\Sigma$ satisfies

$$
\partial_{\Sigma} p(D)=\frac{1}{2} \partial_{-} p(D)\left(1-r_{B}^{\prime}\left(\theta_{D}\right) \lambda(D)\right),
$$

which is exactly the $(S 2)$ condition. Thus we have the compatibility conditions for the mixed initial-boundary value problems, which ensure the local existence of solutions around $D$.

[Uniform $C^{1}$ estimate of $W$ ] Let us consider the uniform estimate of $W$. We note that $\partial_{+} p=0$ in the simple wave zone $R$ since

$$
\partial_{-} \partial_{+} p=q\left(\partial_{-} p-\partial_{+} p\right) \partial_{+} p
$$

with the zero initial condition of $\partial_{+} p$ along the positive characteristic $\Gamma_{2}$. In order to show $\partial_{-} p>0$ in the simple wave zone $R$, let us consider the decomposition

$$
\partial_{+} \partial_{-} p=q\left(\partial_{+} p-\partial_{-} p\right) \partial_{-} p=-q\left(\partial_{-} p\right)^{2} .
$$

We note that the solution $\partial_{-} p$ of this differential equation with the positive initial condition of $\partial_{-} p$ on $\Sigma$ cannot vanish. Hence $\partial_{-} p>0$ in $R$. Since $\partial_{-} p>0$ in the simple wave zone $R, p$ is increasing along the negative characteristic emitting from $\Gamma_{2}$. In addition, since $p$ is increasing along the shock, we have

$$
p_{4} \leq p \leq p(C),
$$

where $p(C)=r^{2}\left(\theta_{C}\right)$. From (15), $\partial_{-} p$ is decreasing along the positive characteristic in $R$, so

$$
0<\partial_{-} p \leq \max _{\Sigma} \partial_{-} p
$$

in $R$. Thus the uniform estimate of $\left(\partial_{+} p, \partial_{-} p, p\right)$ is obtained. Now it is clear that $\partial_{-} \partial_{+} p$ and $\partial_{+} \partial_{-} p$ are uniformly bounded from the decomposition. It 
remains to get the estimates of $\partial_{+} \partial_{+} p$ and $\partial_{-} \partial_{-} p$. First, let us consider the estimate of $\partial_{-} \partial_{-} p$. By decomposition (6),

$$
\begin{aligned}
\partial_{-} \partial_{+} \partial_{-} p= & \partial_{-} q\left(\partial_{+} p-\partial_{-} p\right) \partial_{-} p+q\left(\partial_{-} \partial_{+} p-\partial_{-} \partial_{-} p\right) \partial_{-} p \\
& +q\left(\partial_{+} p-\partial_{-} p\right) \partial_{-} \partial_{-} p
\end{aligned}
$$

where

$$
\partial_{-} q=\frac{8 r p^{2} \sqrt{r^{2}\left(r^{2}-p\right) / p}-4 r^{2}\left(r^{2}-p\right) \partial_{-} p+4 p r^{2} \partial_{-} p}{\left(4 p\left(r^{2}-p\right)\right)^{2}} .
$$

On the other hand, we have the following identity

$$
\partial_{-} \partial_{+}\left(\partial_{-} p\right)-\partial_{+} \partial_{-}\left(\partial_{-} p\right)=\frac{\partial_{-}\left(\lambda_{+}^{-1}\right)-\partial_{+}\left(\lambda_{-}^{-1}\right)}{\lambda_{-}^{-1}-\lambda_{+}^{-1}}\left(\partial_{-} \partial_{-} p-\partial_{+} \partial_{-} p\right)
$$

by direct computations, where $\lambda_{ \pm}= \pm \lambda$. The two relations (16) and (17) yield

$$
\begin{aligned}
\partial_{+}\left(\partial_{-} \partial_{-} p\right)= & \left(\frac{\partial_{-}\left(\lambda_{+}^{-1}\right)-\partial_{+}\left(\lambda_{-}^{-1}\right)}{\lambda_{+}^{-1}-\lambda_{-}^{-1}}+q\left(\partial_{+} p-2 \partial_{-} p\right)\right) \partial_{-} \partial_{-} p \\
& -\frac{\partial_{-}\left(\lambda_{+}^{-1}\right)-\partial_{+}\left(\lambda_{-}^{-1}\right)}{\lambda_{+}^{-1}-\lambda_{-}^{-1}} q\left(\partial_{+} p-\partial_{-} p\right) \partial_{-} p+\partial_{-} q\left(\partial_{+} p-\partial_{-} p\right) \partial_{-} p \\
& +q^{2}\left(\partial_{-} p-\partial_{+} p\right) \partial_{+} p \partial_{-} p,
\end{aligned}
$$

where

$$
\frac{\partial_{+}\left(\lambda_{-}^{-1}\right)-\partial_{-}\left(\lambda_{+}^{-1}\right)}{\lambda_{+}^{-1}-\lambda_{-}^{-1}}=\frac{r^{2}}{4 p\left(r^{2}-p\right)}\left(\partial_{+} p+\partial_{-} p\right) .
$$

Thus the integrals along the positive and the negative characteristics give uniform estimates of $\partial_{-} \partial_{-} p, \partial_{+} \partial_{+} p$. Therefore, we can conclude that we have a uniform $C^{1}$ estimate of $W$ up to the end of the transonic shock except for the end point $C$.

Remark 2.4. We see that the second condition (10) of (S2) follows from (14) in order to get the monotone increasing property of $p$ along the shock $\Sigma$, particularly at the point $D$.

Next, we consider the existence of solutions in the region $S$, called a semihyperbolic patch, bounded by $\Gamma_{1}$ and $\Gamma_{3}$. Since we have $\partial_{+} p>0$ and $\partial_{-} p>0$ at the point $B, \partial_{+} p>0$ along $\Gamma_{3}$. By Lemma 2.3 , we see that $\partial_{-} p>0$ along the positive characteristic curves in the whole region of $R$, so we extend this property continuously to the sonic curve formed by points $A$ and $C$. Thus we now consider a Goursat boundary value problem in the region bounded by two characteristics $\Gamma_{1}$ and $\Gamma_{3}$, and let the values of $p$ and $\partial_{-} p$ on $\Gamma_{3}$ be the limit values of $p$ and $\partial_{-} p$ from the side of $R$, respectively. So the condition on $\partial_{-} p$ along $\Gamma_{3}$ is $\partial_{-} p>0$.

Now we can obtain the local existence of solutions in the region $S$ of the system (8) at $B$ by a similar compatibility condition of Goursat boundary value problems as in $[7,10]$. Here we omit the proof as it is similar to [6]. 
Lemma 2.5. There exists a smooth local solution to the system (8) at B.

Generally, for the global existence of solutions in the region $S$, we need the maximum principle of solutions. Here we provide a new and clearer approach than in $[6]$.

Lemma 2.6 (Suggested by Tianyou Zhang). For a smooth solution $p$ in the hyperbolic domain $S$, the maximums of $\partial_{-} p, \partial_{+} p$ in $S$ are dominated by the boundary values on $\Delta:=\Gamma_{1} \cup \Gamma_{3}$, that is,

$$
\max _{S}\left\{\partial_{+} p, \partial_{-} p\right\} \leq \frac{p_{1}}{p_{4}} \max _{\Delta}\left\{\partial_{+} p, \partial_{-} p\right\} .
$$

Proof. Let us use the following new characteristic decompositions:

$$
\begin{aligned}
& \partial_{+}\left(\frac{\partial_{-} p}{p}\right)=q \frac{\partial_{-} p}{p}\left(\partial_{+} p-\partial_{-} p\right)-\frac{\partial_{+} p}{p} \frac{\partial_{-} p}{p}, \\
& \partial_{-}\left(\frac{\partial_{+} p}{p}\right)=q \frac{\partial_{+} p}{p}\left(\partial_{-} p-\partial_{+} p\right)-\frac{\partial_{+} p}{p} \frac{\partial_{-} p}{p} .
\end{aligned}
$$

We note that $p_{4} \leq p \leq p_{1}$ in $S$. Thus

$$
\max _{S}\left\{\frac{\partial_{+} p}{p_{1}}, \frac{\partial_{-} p}{p_{1}}\right\} \leq \max _{S}\left\{\frac{\partial_{+} p}{p}, \frac{\partial_{-} p}{p}\right\}, \quad \max _{\Delta}\left\{\frac{\partial_{+} p}{p}, \frac{\partial_{-} p}{p}\right\} \leq \max _{\Delta}\left\{\frac{\partial_{+} p}{p_{4}}, \frac{\partial_{-} p}{p_{4}}\right\},
$$

which imply that we only need to prove that

$$
\max _{S}\left\{\frac{\partial_{+} p}{p}, \frac{\partial_{-} p}{p}\right\} \leq M:=\max _{\Delta}\left\{\frac{\partial_{+} p}{p}, \frac{\partial_{-} p}{p}\right\} .
$$

Now there are two cases. Firstly, assume that inside the domain $S, \partial_{-} p / p \geq$ $\partial_{+} p / p$ entirely. From (18), we know that $\partial_{+}\left(\partial_{-} p / p\right) \leq 0$. For a point $z \in S$, we take a positive characteristic emitting from a point $z_{0} \in \Gamma_{3}$ such that

$$
\left.\frac{\partial_{-} p}{p}\right|_{z} \leq\left.\frac{\partial_{-} p}{p}\right|_{z_{0}} \leq M
$$

Secondly, assume that there exists a point $z \in S$ such that $\partial_{-} p / p<\partial_{+} p / p$. Then there is a neighborhood $N$ of $z$ so that $\partial_{-} p / p<\partial_{+} p / p$ holds for every point in $N$. Then we see that $\partial_{-}\left(\partial_{+} p / p\right) \leq 0$ inside $N$. Then a negative characteristic of $z$ meets the boundary point $z_{1} \in \partial N$, and along the characteristic curve, we get

$$
\left.\frac{\partial_{+} p}{p}\right|_{z} \leq\left.\frac{\partial_{+} p}{p}\right|_{z_{1}}=\left.\frac{\partial_{-} p}{p}\right|_{z_{1}}
$$

Now we have two subcases: (a) If we can draw a positive characteristic $Y_{+}$at $z_{1}$ up to $\Gamma_{3}$ without crossing $\frac{\partial_{+} p}{p}$ and $\frac{\partial_{-} p}{p}$ along the characteristic, then

$$
\left.\frac{\partial_{+} p}{p}\right|_{z} \leq\left.\frac{\partial_{+} p}{p}\right|_{z_{1}}=\left.\frac{\partial_{-} p}{p}\right|_{z_{1}} \leq\left.\frac{\partial_{-} p}{p}\right|_{z_{0}} \leq M
$$

where $z_{0}$ is some point in $\Gamma_{3}$. If there is a crossing of $\frac{\partial_{+} p}{p}$ with $\frac{\partial_{-} p}{p}$ at $z_{2} \in Y_{+}$ and there is a neighborhood $S_{1}$ such that $\frac{\partial_{+} p}{p}>\frac{\partial_{-} p}{p}$ again on $S_{1}$, then we have 
to use a negative characteristic at the intersection point $z_{2}$ to use the monotone property, and repeat the above process until we have the maximum principle.

(b) Assume that we cannot find a proper positive characteristic at $z_{1}$. At $z_{1}$, we see that $\partial_{+} p=\partial_{-} p$. So

$$
\partial_{-}\left(\frac{\partial_{+} p}{p}\right)=-\frac{\partial_{+} p}{p} \frac{\partial_{-} p}{p}<0
$$

at $z_{1}$. Then we use a negative characteristic, called $Y_{-}$, for a while or up to and including the boundary $\Gamma_{1}$ such that

$$
\left.\frac{\partial_{+} p}{p}\right|_{z_{1}} \leq\left.\frac{\partial_{+} p}{p}\right|_{z_{3}} \leq\left.\frac{\partial_{-} p}{p}\right|_{z_{3}}
$$

If $z_{3}$ is on $\Gamma_{1}$, then we are finished. If not, we change the moving direction at $z_{3}$ to take a new positive characteristic. If the new positive characteristic reaches $\Gamma_{3}$ without crossing of $\partial_{+} p / p$ and $\partial_{-} p / p$, then we have done. If not, we repeat the above processes. Hence, by repeating the above process continuously, we have

$$
\left.\frac{\partial_{-} p}{p}\right|_{z} \leq\left.\frac{\partial_{+} p}{p}\right|_{z} \leq\left.\frac{\partial_{+} p}{p}\right|_{z_{1}} \leq\left.\frac{\partial_{+} p}{p}\right|_{z_{3}} \leq\left.\frac{\partial_{-} p}{p}\right|_{z_{3}} \leq \cdots \leq M .
$$

Lemma 2.7. Smooth solutions $p$ in the region of $S$ have the following properties:

(1) $r^{2}-p$ is monotone decreasing along any negative characteristic curve starting at a point on the curve $A B$;

(2) $\partial_{-} p=\partial_{+} p$ on the sonic part of the curve $A C$;

(3) The strictly decreasing property of $r^{2}-p$ is preserved along the positive characteristic starting at a point on the boundary $B C$, provided that

$$
p_{1}>p_{4}>M_{0}:=\frac{1}{4}-\sqrt{2}+\sqrt{\frac{3}{2} \sqrt{2}+\frac{33}{16}} ;
$$

(4) Level curves of $r^{2}-p$ are non-characteristic.

Proof. The results of (1), (2), and (4) can be proved as in [6]. Now let us prove (3) only for the simple wave case. Without loss of generality, we assume $p_{1}=1$. Then

$$
\sqrt{p_{4}} \leq r \leq \sqrt{p_{1}}=1 \text { and } p_{4}<p<p_{1}=1 .
$$

Since it is known that $\partial_{+} p / \sqrt{r^{2}-p}>4 \sqrt{p_{4}}$ in [6], we have

$$
r^{\prime \prime}<r+\frac{2\left(r^{\prime}\right)^{2}}{r}-\frac{\partial_{+} p}{2 r^{\prime}}<1+\frac{1}{\sqrt{p_{4}}} \frac{2\left(1-p_{4}\right)}{p_{4}}-2 p_{4} .
$$

Let us choose $p_{4}$ so that $1-2 p_{4}<0$, that is, $1>p_{4}>\frac{1}{2}$. In addition, let us take $p_{4}>0$ so that

$$
p_{4}>\frac{1}{4}-\sqrt{2}+\sqrt{\frac{3}{2} \sqrt{2}+\frac{33}{16}}:=M_{0} .
$$


Then the estimate

$$
r^{\prime \prime}<1+\frac{1}{\sqrt{p_{4}}} \frac{2\left(1-p_{4}\right)}{p_{4}}-2 p_{4}<\left(1-2 p_{4}\right)+\frac{2\left(1-p_{4}\right)}{p_{4}} \sqrt{2}<\frac{1-2 p_{4}}{2}
$$

yields

$$
\left(r^{\prime}\right)^{4}+r^{3} r^{\prime \prime}<\frac{1}{p_{4}^{2}}\left(1-p_{4}\right)^{2}+\frac{1-2 p_{4}}{2}=\frac{2\left(1-p_{4}\right)\left(1-p_{4}-2 \sqrt{2} p_{4}\right)}{2 p_{4}^{2}}<0 .
$$

Hence we have

$$
\partial_{+}\left(r^{2}-p\right)=\frac{2 r r^{\prime}}{\left(r^{2}+\left(r^{\prime}\right)^{2}\right)^{2}}\left(\left(r^{\prime}\right)^{4}+r^{3} r^{\prime \prime}\right)<0 .
$$

Remark 2.8. The same result as in (4) for a rarefaction wave case has been proved in [6]. In the above lemma, we verify the result still holds for simple waves as for rarefaction waves.

Making use of these lemmas, we are led to the following existence of solutions in the interaction region $S$ as in [6].

Lemma 2.9. There exists a smooth solution in the region $S$ of the Goursat boundary value problem to the pressure gradient system (8).

We see that $\partial_{+} p=0$ in the region $R$. On the other hand, $\partial_{+} p>0$ in the region $S \cup \Gamma_{3}$. Then the directional derivative $\partial_{+} p$ has a jump across the curve $\Gamma_{3}$. Thus according to the previous Lemma 2.3 and Lemma 2.9, there exists a smooth solution in the region $R \cup S$ except at the negative characteristic $\Gamma_{3}$. Now letting $\Omega:=\operatorname{int}\left(R \cup \Gamma_{3} \cup S\right)$, we have the following main result.

Theorem 2.10. Let a subsonic solution $\hat{p}$ satisfy that $\partial_{\Sigma} \hat{p}<0$ or $\left|\partial_{\Sigma} \hat{p}\right|$ is sufficiently small. For a given shock $\Sigma$ with conditions $(S 1) \sim(S 2)$ and positive characteristics $\Gamma_{1}$ and $\Gamma_{2}$, there exists a solution $p \in C^{1}(S \cup R)$, which is Lipchitz continuous globally, of the pressure gradient system (8) satisfying the following property in the domain $\Omega$ : the solution is smooth in both the simple wave zone $R$ adjacent to a constant state $p=p_{4}$ and the interaction zone $S$. The directional derivative $\partial_{+} p$, however, has a jump along the negative characteristic curve $\Gamma_{3}$.

Acknowledgment. The author is grateful to Yuxi Zheng and Tianyou Zhang for their interest and helpful discussions.

\section{References}

[1] Z. Dai and T. Zhang, Existence of a global smooth solution for a degenerate Goursat problem of gas dynamics, Arch. Ration. Mech. Anal. 155 (2000), no. 4, 277-298.

[2] J. Glimm, X. Ji, J. Li, X. Li, P. Zhang, T. Zhang, and Y. Zheng, Transonic shock formation in a rarefaction Riemann problem for the $2 D$ compressible Euler equations, SIAM J. Appl. Math. 69 (2008), no. 3, 720-742.

[3] J. Li, T. Zhang, and S. Yang, The Two-dimensional Riemann Problem in Gas Dynamics, Pitman monographs and surveys in pure and applied mathematics 98. Addison Wesley Longman limited, 1998. 
[4] J. Li, T. Zhang, and Y. Zheng, Simple waves and a characteristic decomposition of the two dimensional compressible Euler equations, Comm. Math. Phys. 267 (2006), no. 1, $1-12$.

[5] K. Song, A pressure-gradient system on non-smooth domains, Comm. Partial Differential Equations 28 (2003), 199-221.

[6] K. Song and Y. Zheng, Semi-hyperbolic patches of the pressure gradient system, Disc. Cont. Dyna. Syst. Series A 24 (2009), 1365-1380.

[7] R.-H. Wang and Z.-Q. Wu, Existence and uniqueness of solutions for some mixed initial boundary value problems of quasilinear hyperbolic systems in two independent variables, Acta Sci. Natur. Jilin University 2 (1963), 277-298 (in Chinese).

[8] Y. Zheng, Two-dimensional regular shock reflection for the pressure gradient system of conservation laws, Acta Math. Appl. Sin. Engl. Ser. 22 (2006), no. 2, 177-210.

[9] _ Systems of Conservation Laws: Two-Dimensional Riemann Problems, 38 PNLDE, Birkhäuser, Boston, 2001.

[10] - The compressible Euler system in two space dimensions. Nonlinear conservation laws, fluid systems and related topics, 301-390, Ser. Contemp. Appl. Math. CAM, 13, World Sci. Publishing, Singapore, 2009.

Department of Mathematics

Research Institute For Basic Sciences

Kyung HeE University

SEOUl 130-701, Korea

E-mail address: kyusong@khu.ac.kr 